\title{
La presencia del E/LE en el sector de la enseñanza pública y reĝlada de Letonia
}

\author{
E/AHren presentzia Letoniako irakaskuntza publiko eta arautuaren arloan
}

The presence of S/FL within the public and regulated education in Latvia

\author{
Alla Placinska \\ Universidad de Letonia \\ alla.placinska@lu.Iv \\ https://orcid.org/0000-0003-1867-0566
}

\section{Zenta Liepa}

Universidad de Letonia

zenta.liepa@gmail.com

https://orcid.org/0000-0001-9454-4241

\section{Resumen}

En el presente estudio se analiza la escasa presencia de la lengua española en el sector público y reglado del sistema educativo letón, así como las razones de tal situación. A partir de una investigación con diferentes herramientas como cuestionarios, se ha aplicado la metodología estudio de caso (case of study) (Mayring, 2000; Yin, 2009). Con base en la teoría de la gestión lingüística de Bernard Spolsky (Spolsky, 2009), se demuestra la importancia del papel que juegan las instituciones gubernamentales como gestores lingüísticos en la modificación de las prácticas y actitudes lingüísticas de los estudiantes. Los factores que impiden la consolidación del E/LE (español como lengua extranjera) en el sistema educativo letón son su insuficiente prestigio institucional tanto en el ámbito europeo como nacional y su escasa implantación en el mercado laboral del país. Por el contrario, la diversidad cultural y geográfica que propone la lengua española constituye un factor dominante para su difusión en Letonia.

\section{Palabras clave}

La política lingüística; la enseñanza de E/LE; la actitud lingüística; el estatus; el sistema educativo.

\section{Sumario}

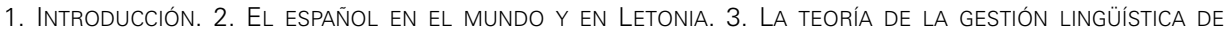
BertRAND SPOlsky. 4. LAS NOCIONES DE ACTITUd LINGÜÍSTICA Y DE PRESTIgIO. 5. MÉTOdO. 5.1. Participantes. 5.2. Instrumento y procedimiento. 6. ANÁLISIS Y RESULTADOS. 6.1. La situación actual en Letonia en relación a la escasa presencia de E/LE en el sector de la enseñanza pública. 6.2. Las actitudes lingüísticas hacia otras lenguas extranjeras y hacia el español. 7. CONCLUSIONES. 


\begin{abstract}
Laburpena. Letoniako hezkuntza sistemako sektore publiko eta arautuan espainierak duen presentzia txikia du aztergai azterketa honek, bai eta egoera horren arrazoiak ere. Zenbait tresnaren bidez egindako ikerketaren bidez, hala nola galdetegiak, kasuen azterketaren bidezko metodologia bat aplikatu da (case study) (Mayring, 2000; Yin, 2009). Bernard Spolskyren hizkuntza kudeaketaren teori-an oinarrituta (Spolsky, 2009), gobernu erakundeek hizkuntza kudeatzaile gisa ikasleen hizkuntza praktika eta jarreren aldaketan duten egitekoaren garrantzia frogatzen da. Honako hauek dira $\mathrm{E} / \mathrm{AH}$ (espainiera atzerriko hitzkuntza gisa) Letoniako hezkun-tza-sisteman sendotzea eragozten duten faktoreak: behar adina prestigio instituzionalik ez izatea bai Europako esparruan bai esparru nazionalean eta bere ezarpen txikia herrialdeko lan merkatuan. Aitzitik, espainiar hizkuntzak proposatzen duen aniz-tasun kultural eta geografikoa faktore erabakigarria da espainiera Letonian hedatzeko.
\end{abstract}

Gako hitzak. Hizkuntza politika; E/AHren irakaskuntza; hizkuntza jarrera; estatusa; hezkuntza sistema.

\begin{abstract}
The article analyses the scarce presence of Spanish within the public and regulated sector of the educational system in Latvia, as well as the causes for this situation. Based on research comprised of different tools, such as questionnaires, the method of case study has been applied (Mayring, 2000; Yin, 2009). The theory of language management by Bernard Spolsky (Spolsky, 2009) demonstrates the importance of the role played by the government institutions as linguistic managers in modifying the linguistic practices and attitudes of students. The factors, which hinder the consolidation of Spanish as a Foreign Language within the Latvian educational system, are the insufficient institutional prestige at the EU and at a national level, as well as the scarce presence of Spanish in the local labour market. On the contrary, the cultural and geographical diversity, proposed by Spanish language, is a dominant factor for its diffusion in Latvia.
\end{abstract}

Keywords: language policy; S/FL teaching; linguistic attitude; status; educational system.

\section{Introducción}

Con la incorporación de Letonia a la Unión Europea (UE) (2004), la importancia de la adquisición de lenguas extranjeras ha crecido enormemente, en especial, entre los jóvenes. A pesar de que el inǵlés goza del privileǵio de ser la lingua franca y su demanda en diferentes ámbitos sigue aumentando (Comisión Europea, 2012a), el multilingüismo, la diversidad cultural, la digitalización y la globalización son fenómenos que, al producir contactos más intensos, directos y multifacéticos, incrementan la motivación para el aprendizaje de lenguas extranjeras. Según la encuesta realizada por el Eurobarómetro en $2012^{1}$, los suecos (32\%), los letones (28\%) y los finlandeses (28\%) aprenden idiomas extranjeros de forma más activa que otros europeos (Comisión Europea, 2012b). Al mismo tiempo, según los últimos datos del Ministerio de Ciencia y Educación de Letonia, solo el 1\% de los alumnos en Letonia estudia E/LE (español como lengua extranjera) en los niveles de educación básica y secundaria. En el sector de la educación superior, el porcentaje es aún más bajo (Liepa, 2019).

A diferencia del promedio europeo, los habitantes de Letonia no incluyen el español en la lista de las lenguas extranjeras que pueden ser de utilidad para su

1 Los datos del año 2012 son los últimos. En la página de la Comisión Europea, no se encuentran datos más recientes. 
desarrollo personal o para el futuro de sus hijos (en la Unión Europea los índices se reparten entre el $16 \%$ en relación con el desarrollo personal y el 14\% en relación con el futuro de sus hijos) (Kibermane y Kḷava, 2016, p. 70). Kibermane y Kḷava (2016) plantean la siguiente pregunta: «¿Qué factores influyen en las preferencias lingüuísticas de los habitantes de un país?». Varios sociolingüuistas letones han dedicado sus investigaciones a temas como la situación sociolingüuística en Letonia, la lenǵua nacional en la educación superior (Baltiņš y Driviete, 2017), la internalización de la educación y la lengua nacional (Druviete y Valdmanis, 2010), las lenguas locales y las lenguas internacionales (Kibermane y Kḷava, 2016) y las actitudes lingüuísticas en Letonia (Baltaiskalna, 2001; Druviete, 2017). Entre los lingüistas españoles, se han abordado en numerosos trabajos temas relacionados con la política lingüuística, la actitud lingüuística y el contacto de lenguas en diferentes ámbitos (político, geográfico, lingüuístico) (Escoriza Morera, 2008; Lodares, 2005; López Morales, 2004; Medina López, 2002; Moreno Fernández,2009; Siǵuán, 2001, etc.). Son numerosas las investigaciones entre los linguüistas anǵlosajones sobre la política y planificación lingüuísticas, lenǵuas de instrucción, contacto de lenguas. Cabe mencionar los trabajos de Fishman (1995), Harris (2007), Lambert (1964), Spolsky (2009). Una de las más interesantes investigaciones en este campo son los estudios de Bernard Spolsky² dedicados a la política y la gestión lingüística. Precisamente la teoría de la gestión lingüuística (language management) propuesta por Spolsky (2009) ha sido aplicada en nuestro análisis de la situación actual en Letonia en relación con la presencia de E/LE en el sector de la enseñanza pública y reǵlamentada.

El objetivo de esta investigación es conocer la presencia del E/LE en el ámbito de la enseñanza de lenguas en Letonia. Las preguntas de investigación que plantea son:

1) ¿Cómo se reǵlamenta la práctica lingüústica en el sector de la enseñanza en Letonia?

2) ¿Cuál es la presencia del E/LE en el sector de la enseñanza?

3) ¿Cuál es la actitud institucional hacia el E/LE en Letonia?

4) ¿Cuál es la actitud de los estudiantes hacia el español como lenǵua extranjera?

La investigación se centró en el sector de la enseñanza pública y reǵlamentada, porque en Letonia es el sector donde se lleva a cabo la política lingüuística del

2 Bernard Spolsky (1932) es, actualmente, profesor emérito de lingüuística en el Departamento de Lenǵua y Literatura Inǵlesas de la Universidad de Bar-Ilan, Israel. Nacido en Nueva Zelanda, estudió en las universidades de Nueva Zelanda y Montreal. A lo largoo de su carrera profesional, trabajó en varias universidades de los EE.UU. Autor de varios libros y trabajos fundamentales en el campo de la sociolingüústica y la lingüuística aplicada, es el fundador de las revistas Applied Linguistics y Language Policy. 
Estado en el ámbito educativo, incluyéndose la enseñanza de lenguas extranjeras. Los colegios, los institutos y las universidades públicas conforman la mayoría de los centros educativos y, además, gozan de más prestigio que la mayoría de los centros privados. Según la cantidad de estudiantes, constituye también el ǵrupo mayoritario. Cabe mencionar que, en muchas ocasiones, ha resultado difícil obtener la información estadística de los centros privados dado que la consideran de carácter confidencial.

\section{El español en el mundo y en Letonia}

Según el último informe del Instituto Cervantes El español una lengua viva. Informe 2020 (Instituto Cervantes, 2020), el español es la lengua que más hablantes nativos del mundo tiene (casi 489 millones, seis millones más que en el año 2019), tras el chino mandarín, y es la tercera lengua en cómputo global de hablantes (dominio nativo + competencia limitada + estudiantes de español), después del inglés y del chino mandarín. Se calcula que, en 2060, la cantidad de los hispanohablantes en los EE.UU. crecerá hasta el 27,5\%; sin embargo, el informe añade, además, que, a pesar de que el número de hispanohablantes seguirá creciendo en las próximas cinco décadas, su peso relativo disminuirá de manera progresiva de aquí a final de siǵlo debido a factores demográficos. Según el mismo informe, más de 22 millones de alumnos estudian español como lengua extranjera. El mayor porcentaje de los estudiantes se ha registrado en los EE.UU. con el $38 \%$; el segundo lugar lo ocupa Brasil con el 28\%; y en el tercer lugar está la UE con el 24\%; el 11\% restante se reparte entre otros países. Siguiendo los datos del Instituto Cervantes, la lengua española es la tercera en internet; casi el 8\% de los internautas se comunica en español. El peso económico del español sigue la misma trayectoria: el español, con el 6,9\%, ocupa la tercera posición tras el inglés y el chino en la clasificación de lenguas en función de su contribución al PIB mundial. En la constelación lingüuística mundial, el español es la segunda lengua más importante en el ámbito internacional, según los datos de la ONU, erigiéndose como la lengua oficial en 21 estados, además de la lengua de trabajo en muchas orǵanizaciones y organismos internacionales (Instituto Cervantes, 2020). También en la Unión Europea el español cierra la lista de las cinco lenguas dominantes: el alemán (72 millones), el inglés (54 millones), el francés (56 millones), el italiano (51 millones) y el español (39 millones) (Baltiņš y Druviete, 2017, p. 100).

A diferencia de la mayoría de los países europeos, la enseñanza del español en Letonia no goza apenas de tradición y es una experiencia relativamente nueva debido a factores políticos, geográficos e históricos. La historia de la enseñanza del español en Letonia es muy breve y tiene sus comienzos a principios del siǵlo XX durante el período de la primera independencia (1918-1940). De 1920 a 1931, 
el profesor A. Spekke, uno de los más conocidos especialistas en lenguas romances adscrito al Departamento de Filología Románica de la Facultad de Filosofía y Letras de la Universidad de Letonia, estableció las bases para el desarrollo de la hispanística en Letonia, dando clases de lengua y literatura españolas, así como de gramática histórica. Después de la pérdida de la soberanía, durante más de cincuenta años, el régimen soviético ejerció el control y el poder en Letonia. Durante esos años, el país fue aislado del mundo exterior y se implementó el principio del Telón de Acero; se restringieron los contactos internacionales y los viajes, y, por lo tanto, los contactos lingüústicos; y se redujo el número de idiomas extranjeros enseñados en las instituciones educativas, así como la cantidad de horas dedicadas a estas asignnaturas. Las políticas del régimen totalitario extendían también a muchas repúblicas soviéticas, incluyendo las repúblicas bálticas, el proceso de la asimilación lingüuística de la población local y la expansión de la lenǵua rusa.

Como consecuencia de los procesos antes mencionados, la lengua rusa llegó a ser la lengúa vehícular en las instituciones gubernamentales y en todas las esferas de la vida social, lo que hizo que se redujese el papel de la lengua letona al ámbito de familia y de las actividades culturales. En el sistema educativo, se admitía solo la enseñanza del inǵlés y el alemán; este último había gozado de una gran tradición en la región báltica y había sido una herramienta de enseñanza a lo largo de varios siǵlos. El privilegio de enseñar la lengua española lo tenían solo las universidades de Moscú, Leningrado (ahora San Petersburgo) y alǵunas más en ciudades soviéticas grandes. En aquel período, el español era una lengua europea exclusiva y exótica. Solo a finales de los años 70 del siǵlo pasado se reimplantó en la Universidad de Letonia la enseñanza de dos lenguas románicas: el francés y el español (prof. M. Rozenberǵa, prof. Z. Dircenko) (Rozenbergá, 1999, p. 61).

Después de la recuperación de la independencia en 1991, el español se incluyó en los proǵramas educativos de los colegios públicos como una asignatura optativa, junto con otras lenguas extranjeras, tales como el italiano, el sueco, el japonés, el chino y el finés. A pesar de que en los últimos veinte años la situación ha cambiado y el español como lengua extranjera se ha introducido en las escuelas y en las universidades, todavía no ha conseguido asentarse. Según los últimos datos del Ministerio de la Ciencia y Educación de Letonia, solo el 1\% de los alumnos en Letonia estudia E/LE (en educación básica y secundaria). En el sector de la educación superior, el porcentaje es aún más bajo (Liepa, 2020).

\section{La teoría de la gestión lingiuística de Bernard Spolsky}

Según Spolsky, la política lingüiística desempeña un papel muy importante en el sistema educativo, por lo que es objeto de interminables discusiones tanto en la comunidad profesional como en la sociedad (Spolskis, 2011, p. 140). El traba- 
jo fundamental de Bernard Spolsky, Gestión lingüística (en original, Language management; en su traducción al letón, Valodas pārvaldība), describe la política lingüuística en diferentes espacios sociales que el autor denomina dominios de colectivos de habla (domain, introducido por D. Fishman) (Spolskis, 2011, p. 18). Cada dominio tiene una red o un espacio de comunicación donde los miembros comparten la variante lingüústica aceptada por la mayoría. Ellos también comparten el mismo tema y lugar (Spolskis, 2011, p. 19). Como ejemplos de dominio, el autor menciona los siguientes: familia, institución educativa, comunidad religiosa, ejército, policía, institución administrativa y empresa. Los participantes del dominio de la familia son los hijos, los padres y otros miembros de la familia, así como personas que trabajan en el servicio doméstico u otro miembro que tiene su papel social en la familia; como ejemplo de lugar, tendríamos la casa y/u otro lugar social donde la familia realiza sus prácticas linguüísticas. El tema en la mayoría de los casos es de carácter informal. Asimismo, hay que tomar en consideración que, en la vida real, cada persona puede ser o es miembro de varios dominios (familia, trabajo, comunidad reliǵiosa) y puede realizar varias prácticas linguuísticas dependiendo del dominio.

El modelo de la política lingüústica elaborado por Spolsky comprende tres componentes independientes pero interrelacionados:

- La práctica lingüústica de los miembros del dominio;

- La actitud lingüuística;

- La gestión lingüuística.

La práctica linguuística es una acción concreta (hablar, utilizar una lengua determinada) que sirve de contexto para la política lingüística. La actitud lingüuística, a veces llamada ideología, está relacionada con los valores y las creencias de los miembros del dominio, y puede resultar muy importante para las comunidades donde la lengua y/o su variante, así como su estatus, poseen un significado especial para la identidad de los miembros. La gestión lingüústica, a su vez, puede ejercer cierta influencia en los miembros del dominio para modificar su práctica lingüuística o sus creencias y actitudes (Spolskis, 2011, p. 21). En cuanto a la terminología, Spolsky indica que prefiere utilizar el término gestión en lugar de la planificación lingüística, que considera ya anticuado. Además, el autor subraya el papel del gestor en todo el proceso, o sea, la acción de una persona o grupo de personas que ejercen el poder con el fin de cambiar o modificar la práctica lingüuística o la actitud de los miembros de un dominio. Por otro lado, Spolsky no recomienda analizar la gestión lingüuística en los casos cuando no se puede identificar al gestor, porque, en este caso, resulta difícil entender los motivos de este.

Generalmente, la política en torno a la gestión lingüuística de la familia suele ser organizada por un miembro con autoridad en ella (o bien el padre o la madre) cuando decide cambiar la práctica lingüuística de otro miembro de la familia 
o determinar qué idioma se debe hablar en ella. Esta práctica se extiende, en términos generales, a los niños, pero también puede afectar al cónyuge o a un miembro que se haya inteǵrado en ella recientemente. Tiene sus raíces en la creencia de que los padres son los responsables de la competencia lingüuística del niño. A esta creencia se vincula el valor de una lengua o variante en particular. Este sistema de valores se basa, generalmente, en experiencias de identidades étnicas o de otro tipo de identidades adquiridas fuera de la familia, así como en creencias sobre la importancia de la pureza lingüústica. Las presiones internas en la familia pueden entrar en conflicto con las presiones externas, lo que evidencia la importancia de analizar la práctica lingüuística en cada dominio por separado, pero siempre debe recordarse que cada dominio está abierto a la influencia de otro dominio más amplio. Ningún hombre es una isla solitaria; ninguna familia es una entidad sociolingüuística cerrada.

El objetivo de la teoría de Spolsky es evaluar las preferencias lingüústicas de los miembros del dominio en relación a los modelos sistémicos adoptados por el colectivo de habla correspondiente. En primer lugar, Spolsky intenta demostrar que las preferencias lingüuísticas de un individuo están determinadas por su visión y aceptación del modelo apropiado para la práctica lingüuística en un dominio particular (Spolskis, 2011, p. 20). Por ejemplo, la madre de una familia no habla igual con su jefe en el trabajo que con sus hijos. Además, ella puede cambiar de lengua y utilizar en el trabajo francés, inglés o árabe (por supuesto, si tiene competencia en estas lenguas). El cambio en la práctica lingüústica (en alǵunos casos se denomina código lingüístico) constituye su elección, a veces casi automática, porque la persona utiliza el lenguaje que considera apropiado para cada dominio. Pero Spolsky demuestra con su teoría que, en muchos casos, esta elección puede ser el resultado de la gestión lingüuística consecuente, consciente y controlada adecuadamente por la parte de los gestores lingüuísticos (Spolskis, 2011, p. 18). Se trata, prácticamente, de una gestión lingüuística organizada y de un modelo en cada y para cada dominio.

Además, cada persona puede tener diferentes funciones: en un dominio, la mujer puede ser el gestor lingüústico y obligar a los hijos hablar con ella en una lengua concreta (para que ellos la aprendan); en otra situación, ser miembro de un dominio donde el gestor lingüuístico le crea una situación cuando ella comprende que puede y debe hablar, por ejemplo, el inglés. No es una obligación legal y oficial; la persona misma toma decisiones, pero estas decisiones son inducidas muchas veces por otros.

Al mismo tiempo, Spolsky señala que, en la elección lingüuística de los miembros del dominio, pueden incidir otros factores, tales como la práctica y la actitud lingüuística (Spolskis, 2011, p. 22). Haberland ha añadido un factor más a este modelo: la necesidad de practicar o hablar una lengua (Haberland, 2011, p. 39). 


\section{Las nociones de actitud linguiuística y de prestigio}

Según la teoría de Spolsky, la expansión de una lengua extranjera en un país determinado depende también de factores tales como la actitud lingüústica, llamada también factor filosófico o ideológico (Spolsky, 2011, p. 21). Ya en el siglo pasado, Saussure destacó que la lengua es un fenómeno social y, por eso, la elección de la lenǵua de comunicación en una situación concreta depende de muchos factores, tanto comunicativos como ideológicos y afectivos (Saussure, 1931, p.10). Entonces, con relación a la enseñanza del español, ¿ será su situación actual el resultado de la gestión lingüística por parte de la administración pública letona o son otros los factores que han ejercido su influencia en la actualidad?

En las últimas décadas, el tema de la actitud lingüuística ha sido abordado en las investigaciones de muchos sociolingüuistas en Letonia. Eso se debe tanto al contacto del letón con otras lenguas como a la preocupación por el estatus de la lengua letona y su función como la lengua oficial y vehicular de Estado. Una de las principales sociolingüuistas de Letonia, la profesora I. Druviete, propone la siguiente definición del término actitud lingüústica. La actitud lingüuística abarca las «peculiaridades de la percepción linguiística de diferentes grupos étnicos, grupos sociales e individuos, la actitud hacia los diferentes idiomas y las medidas tomadas por instituciones estatales o públicas para reǵlamentar la situación lingüística» (Druviete, 1998, p. 99). Otra sociolingüuista letona establece la relación entre una lengua y sus usuarios, comentando que la actitud hacia una variante lingüuística de una lengua coincide con la actitud hacia el hablante que usa esta variante (Baltaiskalna, 2001, p. 11).

En las investigaciones de lingüuistas hispanos, la actitud lingüuística también tiene varias interpretaciones. Por ejemplo, como la reacción que tienen los propios hablantes hacia su lengua y/o a las lenguas habladas en cualquier país multicultural y que se origina por las opiniones, ideas, prejuicios y creencias que los hablantes tengan de ellas (Álvarez, 2009). López Morales expone que las actitudes de un individuo se forman con la suma de tres elementos: lo cognoscitivo (las creencias, las percepciones, los estereotipos), lo afectivo (las emociones y los sentimientos) y lo conductual (tendencia a actuar y reaccionar de cierta manera) (López Morales, 2004, p. 288). A su vez, Moreno Fernández menciona que cada hablante tiene lo que en sociolingüística se denomina conciencia lingüústica. Dependiendo de esa conciencia, los hablantes muestran seguridad e inseguridad lingüísticas. La primera ocurre cuando el hablante considera que sus usos de la lengua coinciden con lo que se considera, según norma, prestigioso o correcto; la seǵunda surge cuando la coincidencia disminuye (Moreno Fernández, 2009).

La noción de prestigio es una de las más importantes en el estudio de las actitudes lingüústicas. Moreno Fernández define el prestiǵio como «un proceso de concesión de estima y respeto hacia individuos o grupos que reúnen ciertas caracterís- 
ticas, y que lleva a la imitación de las conductas y creencias de esos individuos o grupos», es decir, el prestigio es la valoración positiva que atribuyen los hablantes, de forma subjetiva, a una variedad dialectal, a unos usos lingúísticos, a un acento (Moreno Fernández, 2009, p. 187). El prestigío puede entenderse desde dos puntos de vista: (1) como conducta (algo que se tiene) y (2) como actitud (algo que se otorga). Los sociolingüuistas han dado especial importancia al segundo enfoque, razón por la cual han considerado que aquello que el hablante cree correcto es, a su vez, lo que cree más prestigioso, sin olvidar que las normas del prestigio pueden variar de una comunidad de habla a otra (Moreno Fernández, 2009, p. 188).

La actitud lingüuística puede ser positiva, neutral o negativa. La actitud positiva hacia una lengua puede favorecer el uso de esta en detrimento de otra, puede hacer que el aprendizaje de una lengua extranjera sea más satisfactorio, y/o que una variedad sea empleada en contextos más formales o menos formales. En países escandinavos, el tema de las actitudes lingüuísticas se ha convertido en un tema de actualidad en las últimas décadas al evaluarse la expansión del idioma inglés. En Finlandia tiene lugar un gran debate sobre la enseñanza del sueco como segundo idioma obligatorio en las escuelas y la creciente influencia del inǵlés en todos los campos sociales (Druviete, 2016, p. 30).

La profesora Druviete sostiene que la actitud lingüística debe formarse principalmente a través de la educación, porque es esta la que puede influir de manera decisiva en el sistema de valores de la sociedad y luego asegurar su continuidad (Baltiņš y Druviete, 2017, p. 15). Su punto de vista solo confirma la aproximación teórica de la gestión lingüística de Spolsky y la influencia de los gestores lingüuísticos en la elección lingüústica realizada por los alumnos.

\section{Método}

A partir de una investigación (en acción) centrada en la experiencia de Letonia, se aplicó el método del estudio de caso con el fin de llevar a cabo un análisis cualitativo y cuantitativo (case study) (Mayring, 2000; Yin, 2009) sobre la presencia de E/LE en el sector de la enseñanza pública y reǵlada. Para obtener datos sobre la actitud institucional hacia el E/LE, se hizo lectura crítica de la base normativa relacionada con la reglamentación de la enseñanza de lenguas en el ámbito escolar y universitario, cuyos datos son de libre acceso en internet, así como se analizó la información estadística proporcionada por el Ministerio de Ciencia y Educación de la República de Letonia. A su vez, los datos sobre la actitud de los estudiantes hacia E/LE fueron obtenidos aplicando la técnica de encuestas. Con este fin fueron diseñados dos cuestionarios. A continuación, se ofrece la información más detallada sobre las características de los cuestionarios y el procedimiento de la encuesta. 


\subsection{Participantes}

El grupo destinatario (target group) está conformado por jóvenes universitarios tanto de primer ciclo de estudios como de máster. A estos se añaden, además, alumnos de bachillerato.

El perfil de los encuestados en los dos grupos es similar, hablamos de gente joven: el $60 \%$ ronda entre los 18 o 20 años, y el $20 \%$ alcanza los 30 años; la mayoría, el $65 \%$, son mujeres. El primer grupo está formado por estudiantes de bachillerato o de universidades, y el segundo grupo mayoritario está formado también por estudiantes universitarios, tanto por los que solo estudian como por los que ya están trabajando, pero hacen los estudios de máster. En general, los encuestados son gente joven y activa, o bien están comenzando su formación profesional o bien están al comienzo de su carrera profesional. Además, los encuestados, en su mayoría, ya conocen otra lengua extranjera, como el inglés $(94,5 \%)$, el ruso $(57,3 \%)$, y el alemán (15,1\%). Para la mayoría, el español es L2 o L3.

\subsection{Instrumento y procedimiento}

El diseño de los cuestionarios se apoya en la metodología elaborada por la socióloga letona S. Kristapsone (Kristapsone, 2014). Las preguntas formuladas son breves y fáciles de entender y poseen la opción de respuestas múltiples. Cada encuesta se realizó con carácter anónimo y posee cinco preguntas de introducción para determinar el perfil del encuestado (edad, género, educación, lengua materna y la lengua que se habla en la familia) y diez/once preguntas que comprenden las respuestas relacionadas con la percepción afectiva hacia las lenguas extranjeras. El tiempo de cumplimentación de cada encuesta no superaba los cinco minutos y se realizó online utilizando Google Forms. Para evitar recibir cuestionarios incompletos, todas las preguntas se marcaron como obligatorias y el sistema no permitió el envío de la encuestas incompletas. Cada encuesta fue diseñada en conformidad con los distintos grupos de jóvenes. La primera encuesta se aplicó al ǵrupo de jóvenes que no estudian ni saben español con el fin de obtener datos sobre sus prácticas lingüísticas y su actitud hacia las lenguas extranjeras, en general, y hacia el español, en particular. El número de encuestados en este caso es de 444 (Encuesta 1). La segunda encuesta se aplicó al alumnado que estudia el español con el objetivo de evaluar su actitud e interés hacía este idioma y los motivos para estudiarlo. El número de encuestados en este otro caso es de 301 (Encuesta 2). En total, se encuestaron 745 personas. Las respuestas aparecen analizadas y contrastadas en la sección 5.2. dedicada a la actitud linguiústica.

Las encuestas se hicieron en letón para obtener la mayor cantidad de información, y sus resultados principales han sido traducidos al español por las autoras del presente artículo. Las encuestas se hicieron en los meses de la primavera 
de 2020 en la Universidad de Letonia, la Universidad de Medicina de Stradiņš y la Academia de Cultura de Letonia, así como entre diferentes colegios públicos donde se enseña el español, la mayoría de ellos situados en Riga, y fueron realizadas con la ayuda de profesores de lengua.

Los datos obtenidos a través de las encuestas y de la lectura crítica de los actos normativos de estado han sido analizados desde el punto de vista de la teoría de la gestión linguüística de Spolsky (Spolsky, 2009).

\section{Análisis y resultados}

\subsection{La situación actual en Letonia en relación a la escasa presencia de E/ LE en el sector de la enseñanza pública}

A continuación, se intenta de explicar las razones de la situación actual en Letonia en relación a la escasa presencia de E/LE en el sector de la enseñanza pública a través de la teoría de la gestión lingüística de Spolsky. Los gestores lingüuísticos en el sector de la enseñanza pública son directores de centros de enseñanza. El Ministerio de Educación es también un gestor lingüístico, y es el gestor superior, porque además del poder ejecutivo, posee la iniciativa legislativa. El análisis de múltiples documentos normativos demuestra que el sector educativo en Letonia está muy bien reǵlamentado por una seria de leyes y documentos normativos los cuales dejan poca posibilidad de iniciativa a los directores de centros en el campo de la gestión linguiuística. Según la socióloga letona I. Druviete, el sistema educativo es un sistema jerárquico y lineal. La práctica lingüuística en uno de los niveles, por ejemplo, el universitario, afecta a las prácticas lingüuísticas en otros niveles del sistema como la educación secundaria (Druviete y Valdmanis, 2010, p. 92). Esto coincide totalmente con la aproximación teórica de Spolsky: «En la mayoría de las sociedades el colegio es la primera institución que realiza una política lingüuística dando prioridad a un idioma en particular. Desde el momento en que un niño ingresa en la escuela, sus hábitos lingüuísticos se modifican ${ }^{3}$ (Spolskis, 2011, p. 140).

Los principales documentos políticos relativos a la educación primaria y secundaria son los siguientes:

- Reǵlamento 747 del Consejo de Ministros sobre estándares en la educación básica y ejemplos de programas de educación básica (Legal Acts of the Republic of Latvia, 2021).

- Reǵlamento del Consejo de Ministros 416 sobre los estándares en educación secundaria general y ejemplos de programas de educación secundaria general (Legal Acts of the Republic of Latvia, 2021).

\footnotetext{
3 La traducción de las citas y referencias del letón al español es de las autoras del trabajo.
} 
- Proyecto Escuela 2030 del Ministerio de Ciencia y Educación (Ministerio de Ciencia y Educación de la República de Letonia, 2021).

En estos documentos políticos está reǵlamentado paso a paso todo el proceso educativo, incluyendo el campo de la enseñanza de lenguas extranjeras. Según el proyecto Escuela 2030, la adquisición obligatoria de dos lenguas extranjeras se va a mantener. Todos los alumnos deben aprender una de las lenguas de la Unión Europea desde el nivel cero al menos hasta el nivel B2 (nivel óptimo) y la segunda lengua extranjera desde el $3 .^{\circ}-4{ }^{\circ}$ grado hasta al menos el nivel B1 (nivel general). A diferencia de los programas anteriores, el aprendizaje de la primera lengua obligatoria empezará a partir del $1 .^{\circ}$ grado. Cada centro está autorizado también a ofrecer a los estudiantes una tercera lengua extranjera como asignatura de libre elección en la que, dependiendo del número de horas dadas, los estudiantes alcanzarán niveles A2 / B1 (nivel general) (Ministerio de Ciencia y Educación de la República de Letonia, 2021).

Cabe mencionar que la normativa no obliga a elegir el inglés como la primera lengua extranjera, pero la mayoría de los alumnos optan por aprender esta lengua como el primer idioma, aunque el primer idioma extranjero también puede ser el alemán, el francés, etc., como lo es en respectivos liceos especializados (Liceo Francés, Liceo Alemán).

El número de segundas lenǵuas extranjeras que se pueden aprender en las escuelas de educación general de Letonia supera las veinte. Además de los idiomas antes mencionados, también se ofrece el estonio (Escuela Primaria de Estonia de Riga), el italiano (Escuela Secundaria de Culturas de Riga), el chino (Liceo de Jekabpils y Escuela Secundaria de Culturas de Riga), el japonés (Escuela Secundaria de Culturas de Rigá), el danés (Liceo Nórdico) y el noruegoo (Escuela Secundaria de Ķenǵaraǵs de Riǵa, Liceo de Pārǵauja, Colegio Nórdico), etc.

Desde el punto de vista teórico, el español podría enseñarse como la primera y la segunda lengua extranjera obligatoria, aunque, en realidad, se enseñanza básicamente como la tercera lengua (asiǵnatura optativa) y, en muy pocos coleǵios, como la L2. Una de las razones por las que el español no se ofrece como la primera lengua extranjera es su ausencia de los exámenes de selectividad. Los exámenes los elabora y orǵaniza el Ministerio de la Ciencia y Educación de la República de Letonia (Legal Acts of the Republic of Latvia, 2021). Estos exámenes son obligatorios para poder culminar satisfactoriamente la secundaria y el bachillerato. Están elaborados solo para el inglés, el francés, el alemán y el ruso; esto quiere decir que el español puede ser enseñado solo como la segunda lenǵua obligatoria o como la tercera con carácter de lengua optativa. Además, los Reǵlamentos del Consejo de Ministros 416 admiten los Diplomas DELE como equivalentes para el examen de selectividad. En Riga hay un Centro de Exámenes DELE, lo cual ofrece esta posibilidad a los estudiantes, pero existen dos inconvenientes: (1) los precios de los exámenes DELE son bastante altos y (2) los diplomas, en el mejor de los casos, llegan medio año después del examen. 
Según los datos estadísticos proporcionados por el Ministerio de la Ciencia y Educación en el año escolar de 2018/2019, el español se podía aprender en dieciocho colegios de la educación general básica (a partir del 6. grado) y en 2019/2020, en 16 coleǵios. El número de alumnos de español en estos coleǵios varía entre 1436 en el 2018/2019 y 1176 en el 2019/2020. Según los datos proporcionados por el Ministerio en el mes de abril de 2021, el español se ofrece en trece instituciones educativas de la educación general secundaria en Letonia, de las cuales solo cuatro están en la provincia y el resto se concentra en Riga, la capital. Debido a la ausencia de estadísticas de libre acceso, los datos han sido proporcionados por la funcionaria del Ministerio vía correo electrónico. Es de notar el descenso de las cantidad de centros (de dieciocho en 2019 a doce en 2021), así como la cantidad de alumnos. Esto se debe, sobre todo, al descenso demográfico y a la emigración, y no altera radicalmente la situación como tal en el sector educativo. En la mayoría de los colegios, el español se enseña como la tercera lengua, es decir, como una asignatura optativa. (O. Arkle, comunicación personal, 20 de agosto de 2020). Cabe mencionar que hay demanda de profesores de español en los coleǵios, pero es difícil cubrir las vacantes ofrecidas debido a una remuneración insuficiente y a una carǵa académica incompleta, puesto que, en la mayoría de los casos, la forma de trabajo es por horas.

En el sector de la educación superior, la enseñanza de las lenguas y la enseñanza de las lenǵuas extranjeras está regulada por la Ley de Educación Superior en la República de Letonia. Entre las prioridades de la política en este sector, está la internacionalización de las universidades, la creación de programas conjuntos con otras universidades de los países miembros de la UE y la movilidad del personal académico (Latvijas Vēstnesis, 2021).

El artículo 56 de la Ley establece que la lengua de instrucción es el letón, y que las lenguas extranjeras se utilizan en los programas de la UE, así como en los programas que son fruto de proyectos internacionales (por ejemplo, Erasmus). También se imparten en idiomas extranjeros los programas de idiomas y los estudios culturales. Por lo tanto, cada institución de educación superior tiene derecho a decidir sobre los programas de estudios que se implementan y sobre la(s) lenǵua(s) de instrucción.

Hay programas de lenguas extranjeras donde una parte de las asignaturas se imparte en letón y la otra en un idioma extranjero (en diferentes proǵramas de Filología o estudios de lenguas extranjeras) y proǵramas que se realizan en lenguas extranjeras, básicamente, en inglés (Programa de Estudios Europeos, programas de Administración de Empresas, de Comercio y Marketing, etc.).

Pero la lengua española como lenǵua de instrucción solo se utiliza en dos centros: en la Academia de Cultura, en el subprograma de grado Relaciones Interculturales Letonia-España, y en la Universidad de Letonia, en los subprogramas de Lenguas y Negocios y Filología Francesa. En la Academia de Cultura, el programa abre la matrícula solo una vez cada cuatro años. El programa de Relaciones In- 
terculturales en la Academia funciona desde 1995. Según la información proporcionada por el Departamento de Estudios, la cantidad total de egresados equivale a 101 estudiantes (A. Karlsone, comunicación personal, 7 de abril de 2021). Los alumnos estudian el español desde el nivel cero y llegan hasta el nivel B2, según el Marco Común Europeo.

En la Universidad de Letonia, en el nivel de máster, se realiza el subprograma de Lenguas y Culturas Románicas, que es prácticamente el único programa de estudios superiores de español y de la cultura respectiva. Para realizar los estudios en español, es necesario matricularse en el módulo español (paralelamente, hay módulos en francés e italiano, según la lengua de instrucción). El programa se abrió en el 2011, y fue completado por cuarenta estudiantes. El español se enseña también en otros proǵramas como L2 o L3 (asignatura optativa). De acuerdo con la información recibida del Departamento de Lenguas Románicas, durante el año académico 2019-2020, la cantidad de estudiantes de español en la Universidad de Letonia fue de 289 alumnos (A. Šnipke, comunicación personal, 15 de junio de 2021). La Universidad de Letonia sigue siendo el centro con la mayor cantidad de estudiantes del español. No obstante, en algunas universidades de provincias se enseña español también (la Universidad de Liepaja, la Universidad de Daugavpils).

Los datos demuestran el interés por parte de los estudiantes hacia el español como LE; sin embargó, a día de hoy, la administración de la Universidad, como gestor lingüuístico, no ha tomado la decisión de abrir un programa o un subprograma en el grado de Filología Hispánica o de Estudios del Español. El arǵumento que se esǵrime a este respecto con mayor frecuencia es la poca presencia del español en la educación secundaria y, como consecuencia, el bajo nivel de competencia lingüuística y la baja demanda del español en el mercado laboral de Letonia y de otros países de la región báltica.

\subsection{Las actitudes lingüuísticas hacia otras lenǵuas extranjeras y hacia el español}

La actitud de la población letona hacia las lenǵuas extranjeras es muy positiva. Según la encuesta realizada por la Agencia Nacional de la Lengua Letona, los letones se diferencian del ciudadano medio de la UE por el hecho de que la población de Letonia demuestra un gran apoyo a la idea y a los objetivos del multilingúiismo. El 91\% de los letones encuestados cree que todos los ciudadanos de la Unión Europea deberían hablar al menos un idioma extranjero, además de su lengua materna, lo que representa un índice promedio mayor en la UE (84\%) comparado con la media de la UE (72\%). Como la mayoría de los letones puede comunicarse en varios idiomas, entre ellos el letón y el ruso, como mínimo, el $97 \%$ cree que los idiomas extranjeros son útiles; este es también un indicador más alto que la media de la UE (88\%) (Kibermane y Kḷava, 2016, p. 66). Los encuestados creen que los idiomas más útiles para su desarrollo personal y para el futuro 
de sus hijos son el inglés (para el desarrollo personal -el 72\%, para los niños-, el $92 \%$ ), el ruso (para el desarrollo personal -el 50\%, para los niños-, el 48\%) y el alemán (para el desarrollo personal -el 17\%, para los niños-, el 21\%); raramente se mencionan otros idiomas. Y si llevamos a cabo una comparación, los ciudadanos de la UE también consideran en promedio que el inǵlés (el 67\% para el desarrollo personal, el 79\% para niños) y el alemán (el 17\% para desarrollo personal, el $20 \%$ para niños) son los más útiles para su desarrollo personal, así como para sus hijos, seguido por el francés (para desarrollo personal -el 16\%, para niños-, el 20\%) y el español (para desarrollo personal -el 14\%, para niños-, el 16\%). En la categoría de los idiomas útiles para los niños, también se incluye el chino (el 14\%); a este respecto, la población letona, a diferencia de los representantes de otros países de la UE, no lo menciona en absoluto (Kibermane y Kḷava, 2016, p. 70). ¿Por qué, a diferencia de la media europea, los habitantes de Letonia no han incluido la lengua española en la lista de las lenguas extranjeras que pueden ser de utilidad? Como Kibermane y Kḷava (2016) en su investigación no analizan las razones de tal situación, el tema de la actitud hacia el español ha sido incluida en la presente investigación y, como consecuencia, en las encuestas realizadas a los estudiantes. A continuación ofrecemos los resultados de las encuestas.

A la pregunta «¿Cuáles son las tres razones para aprender el español?», los que no están estudiando esta lengua dan como la principal razón para estudiarla que ofrece mejores oportunidades en el mercado laboral, que el español es la segunda lengua más hablada en el mundo, y que brinda la posibilidad de establecer nuevos contactos. Las respuestas indican que los encuestados son bastante praǵmáticos, valoran el estatus internacional de la lenǵua española, su peso económico y las ventajas en el mercado laboral, y la consideran una herramienta eficaz en la comunicación.

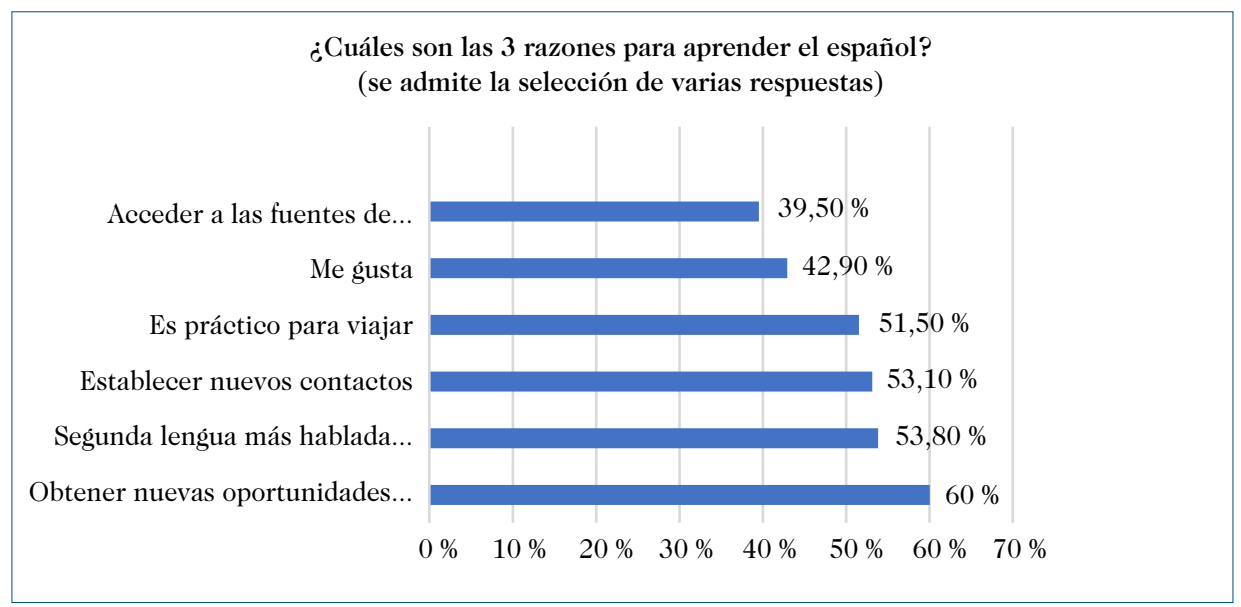

Figura 1. Razones para aprender el español del grupo 1. 
La misma actitud demuestran los encuestados en el segundo grupo, que está conformado por los que estudian español. A la pregunta «¿Qué te aportan los estudios del español?», el $57 \%$ indica que proporcionan mejores oportunidades en el mercado laboral. Otro índice muy alto es la importancia que los estudiantes adjudican al conocimiento de las lenguas. Además, la mayoría de ellos (el 70,3\%) responden a la próxima pregunta afirmando que van a seguir estudiando español.

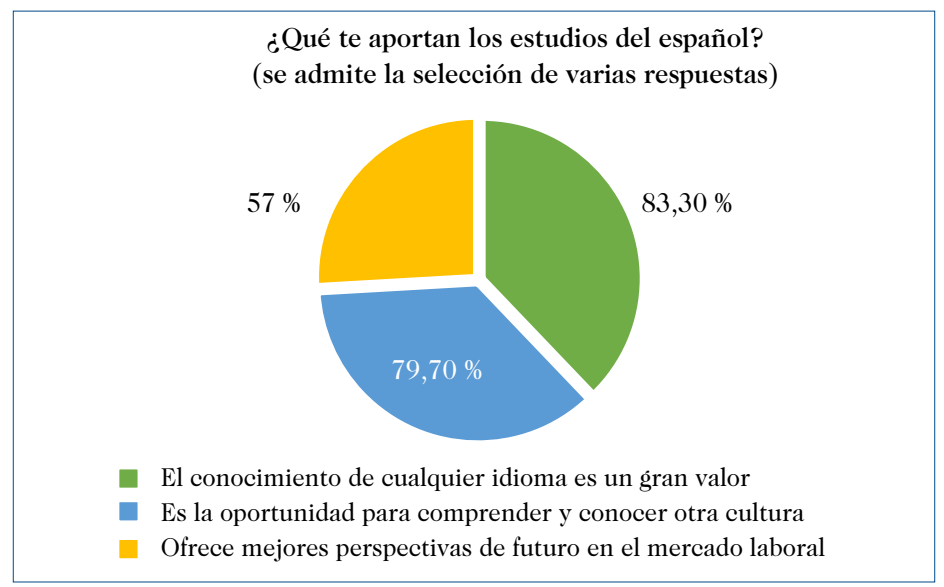

Figura 2. ¿Qué te aportan los estudios del español?

Las respuestas que se dan a la pregunta «¿Por qué empezaste a estudiar el español?» revelan que la mayoría lo ha hecho simplemente porque ha aprovechado la oportunidad que les ha propuesto el centro donde estudian. Eso significa que la elección de estudiar el español se ha hecho sin una gran motivación, simplemente porque se les dio dicha oportunidad y rechazaron estudiar francés o alemán frente a la lengua española. Un $10 \%$ reconoce abiertamente esta ausencia de motivación. Los que contestaron que simplemente querían estudiar una nueva lengua tampoco hablan de motivación, lo que demuestra que incluso entre los que estudian el español lo han hecho en gran medida sin tener al principio ninguna motivación para hacerlo. Pero el aprendizaje de la lengua, o mejor dicho, la posibilidad de hacerlo, ha ayudado a muchos después a encontrar una motivación y cierto interés por la lengua. Aquí se ve la importancia del papel que tienen los gestores lingüísticos en la difusión de una lengua y en la formación de la actitud hacia ella. Si la institución no les hubiera propuesto esta oportunidad de aprender español, tendríamos una menor cantidad de estudiantes y, por supuesto, menos personas interesadas en continuar estudiándolo. Se aprecia muy bien cómo los gestores linguiústicos han modificado y han cambiado la actitud y las prácticas lingüuísticas de los encuestados. 


\section{La razón por la que estás aprendiendo español}

(se admite la selección de varias respuestas)

Estudio sin ninguna motivación

Me lo aconsejaron amigos, conocidos...

Estudio español por el interés cultural...

En relación con mis planes en el futuro

Simplemente quería aprender un idioma...

Por mi propio interés personal

Aproveché de la oportunidad en mi...

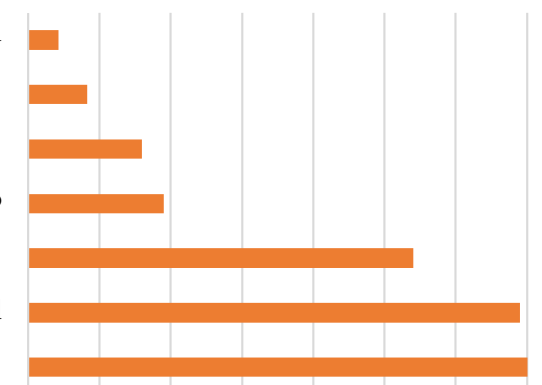

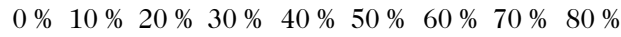

Figura 3. Razones para aprender el español del grupo 2.

Las respuestas a la pregunta « ¿ Va a crecer el interés por la lengua y la cultura españolas en Letonia?» revelan un estatus inestable del español en el mercado de las lenǵuas extranjeras en Letonia y, como consecuencia, un futuro preocupante. Son las respuestas del primer grupo de encuestados, es decir, de los que no estudian español. Solo un $11 \%$ de ellos están seguros del resultado positivo. Una gran parte lo considera probable, pero no está segura, y, lo que es peor: un $40 \%$ está seguro de lo contrario. Esto se explica considerando el insuficiente prestigio del español y su cultura en nuestro país. Los encuestados no ven la necesidad de dedicar su tiempo a aprender español debido al hecho de que su posición en el mercado letón y, en general, en el mercado europeo, no está muy clara.

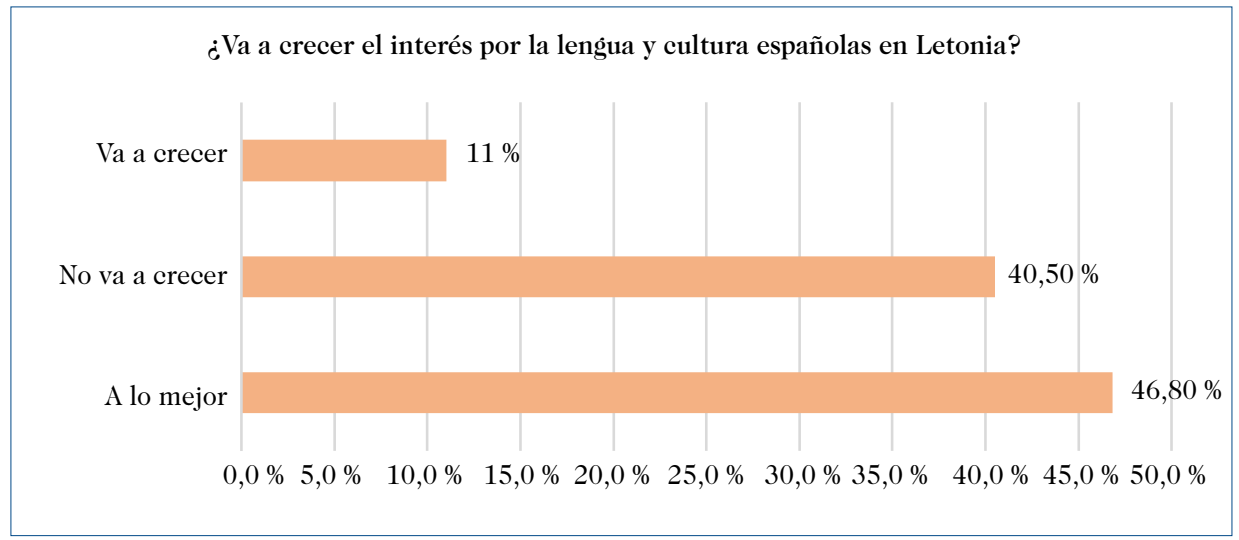

Figura 4. Interés por la lengua y la cultura españolas en el futuro. 
Preocupantes son las respuestas del segundo grupo, es decir, de los que están estudiando español. Un alto porcentaje de los que no están seguros si el español tiene potencial de crecimiento en el futuro confirma la inseguridad lingústica que tienen los estudiantes respecto al español y su futuro en Letonia. Como algo positivo podemos comentar que una gran parte de los que están estudiando la lengua están seguros de su crecimiento en el futuro.

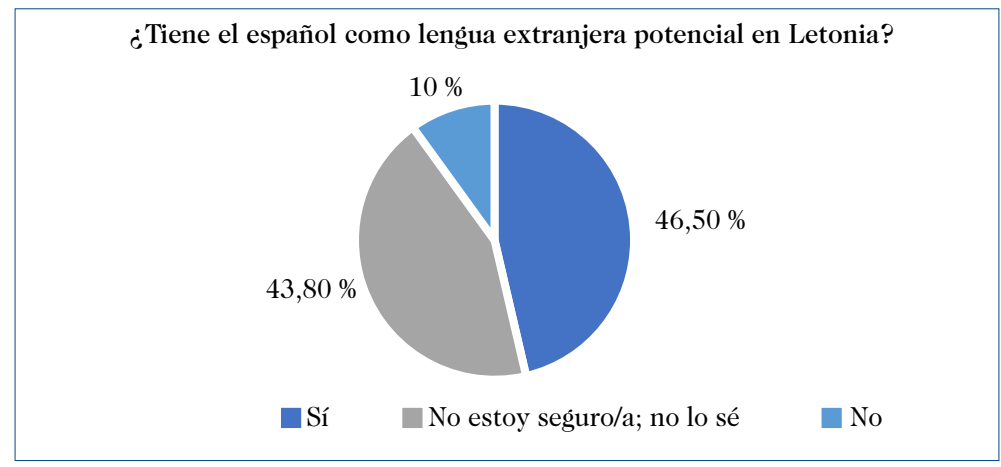

Figura 5. Previsiones sobre el futuro del español.

Las respuestas a la pregunta «¿Cuál debería ser el estatus de E/LE en los colegios?» confirman la inseguridad linguística hacia el español que tiene la mayoría de los encuestados. En el caso de los que no estudian español, el número de los que considera que el español debe enseñarse solo como asignatura optativa alcanza casi al 95\%, y casi el 5\% de los encuestados considera que ni vale la pena incluirla en la oferta de estudios. Incluso entre los que ya están estudiando la lenǵua española, las respuestas son muy similares.

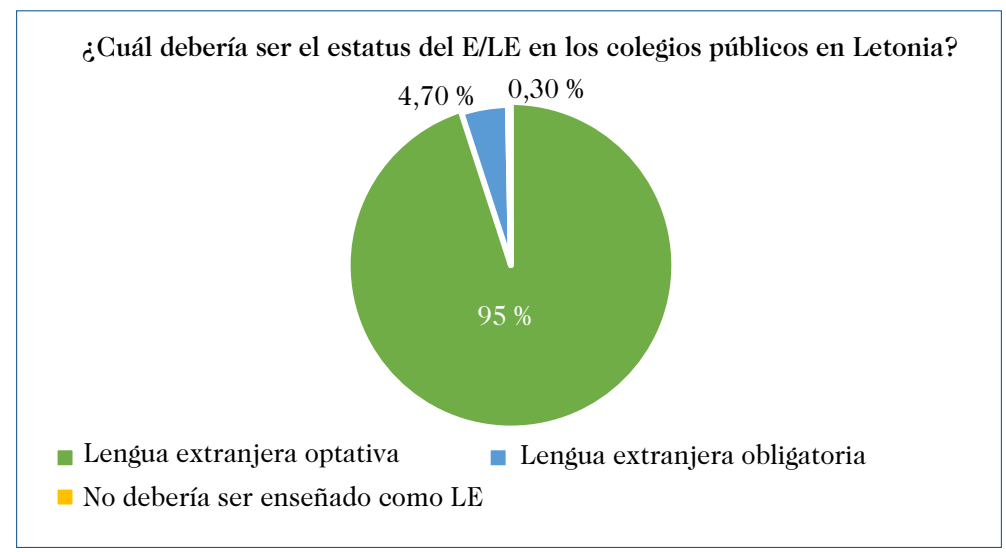

Figura 6. Opinión sobre el estatus del español como LE en los colegios. 
$\mathrm{Al}$ grupo de los alumnos que no estudian el español se le ha pedido indicar las lenguas que deberían aprender los niños. Como se ve en las respuestas, domina el inǵlés, seguido por el ruso, el alemán y el español. Con relación al promedio europeo, se destaca la ausencia del chino en las respuestas. Esto permite concluir que el futuro del español en Letonia tiene perspectivas más positivas que negativas, y que la actitud hacia la lengua está cambiando, aunque de manera muy lenta. Este diagrama también demuestra la importancia del factor de la actitud lingüústica. El francés, que tiene en Letonia un apoyo importante y estable por parte de los gestores lingüústicos a lo largoo de muchos años, se sitúa por detrás del español, con un $14 \%$, y por eso no ha sido incluido en el gráfico.

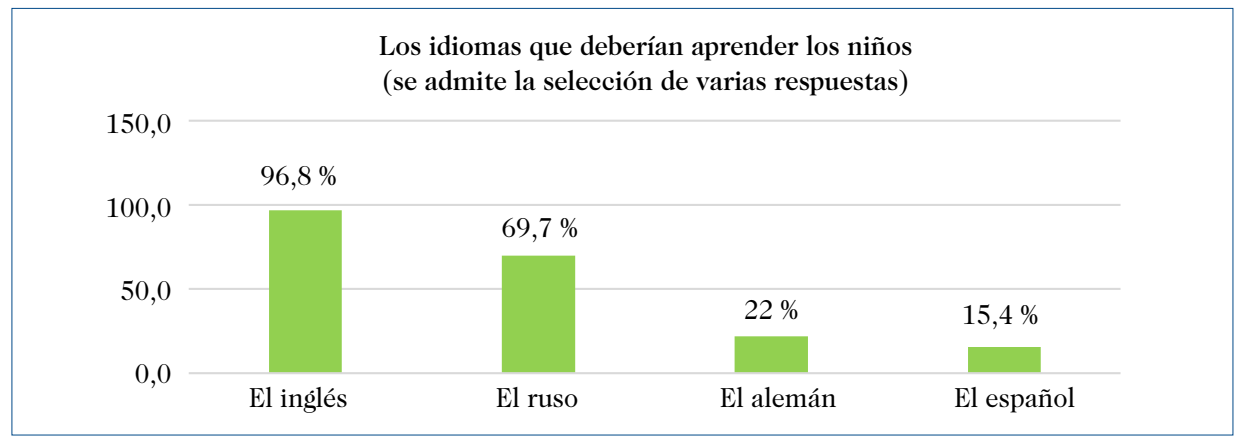

Figura 7. Los idiomas que deberían aprender los niños.

Cerrando el apartado de la actitud lingüuística, es importante destacar que las encuestas han aportado información tanto cuantitativa como cualitativamente sobre el tema de la enseñanza del español en Letonia. La fuerza de la tradición, el estatus y el prestigio de una lengua constituyen los factores importantes que determinan la conducta de los gestores de la política lingüuística. A su vez, los aspectos culturales, la demanda en el mercado laboral y el turismo son los factores que crean una actitud positiva hacia el español entre la población joven en Letonia.

\section{Conclusiones}

A pesar de que en los últimos diez años la situación ha cambiado y el español como lengua extranjera se ha introducido en las escuelas (en la educación primaria y secundaria) y en las universidades, todavía no ha conseguido asentarse positivamente como hubiera sido lo deseable para la oferta de la lengua española.

El español no ha alcanzado el estatus de una lengua obligatoria en el dominio de los colegios, ni ha ampliado su presencia en los programas universitarios a pesar de su importancia internacional (el dominio universitario). Tanto los gestores lin- 
güísticos de diferentes niveles (Ministerio de la Educación, administración universitaria, directores de colegios etc.) como los miembros de los respectivos dominios han demostrado un alto índice en cuanto a la noción de inseguridad lingüística respecto a la necesidad de saber y aprender el español en el futuro, lo cual ha llevado a la modificación de las prácticas y actitudes linguiuísticas del alumnado. Se puede concluir que la importancia ǵlobal del español cede a la escasez de importancia local (el español no tiene el mismo estatus y el prestigio que tienen a nivel europeo el inǵlés, el francés y el alemán). La gestión lingüuística en el campo de las lenguas extranjeras demuestra la fuerza de la tradición en la selección de las lenguas, el apoyo al inglés como lengua dominante en el campo de las lenǵuas extranjeras y el poco interés hacia el español como un jugador de peso en esta área. En los colegios donde los gestores han propuesto los estudios del español, la mayoría de los alumnos ha cambiado su actitud hacia el español y piensa seguir estudiándolo, lo que ha llevado, a su vez, al cambio en sus prácticas lingüuísticas. Es bastante difícil reemplazar el inǵlés, el alemán, el ruso o el francés enseñados tradicionalmente por otros idiomas europeos $\mathrm{u}$ orientales, porque el Estado, como gestor principal, no ha visto la necesidad de hacerlo. La prevalencia y la popularidad del idioma español en Letonia no es tan alta como podría ser tomando en cuenta su posición demográfica, económica e importancia cultural. La población joven tiene una actitud muy positiva hacia el español y la cultura hispana, valora su importancia como vehículo de comunicación, pero, debido a la inseguridad lingüuística y la actitud pasiva por la parte de los gestores, no está dispuesta a alterar sustancialmente sus prácticas lingüuísticas. El factor importante para la gestión lingüuística es el estatus de la lengua, el cual depende de muchos factores tanto objetivos (peso económico, factores demográficos, institucionales, factores geográficos y políticos) como subjetivos (creencias lingüísticas, actitud, tradición).

Asimismo, es importante el apoyo institucional por parte de España. De momento, no existe un Instituto Cervantes en ninguno de los países bálticos; sin embargo, se valora muy positivamente la posibilidad de colaborar con los lectores de español enviados por la Agencia Española de Cooperación Internacional para el Desarrollo [AECID] que trabajan en la Universidad de Letonia y en la Academia de Cultura de Letonia. Los cambios en la gestión linguüística en el campo de la enseñanza de lenguas extranjeras en Letonia se pueden esperar cuando haya cambio en el estatus de la lengua española tanto a nivel institucional europeo como a nivel local. En la actualidad, el interés hacia la diversidad cultural y geográfica que propone la lengua española constituye el factor dominante para su difusión en Letonia. Este país, con sus dos millones de habitantes, no es un jugador ǵlobal en el mercado de lenguas, pero forma parte y participa en muchos procesos internacionales, con lo cual los aspectos que han sido analizados en el presente artículo puedan servir de indicadores para marcar las tendencias a nivel global ${ }^{4}$.

4 La preparación del presente artículo ha contado con el apoyo del proyecto Nr.VPP-IZM-2018/2-0002 dentro del Programa Nacional de Investigaciones de Letonia. 


\section{Referencias}

Álvarez, A. (2009). Sobre la construcción discursiva del país: actitudes lingüuísticas en Venezuela. Presente y Pasado. Revista de Historia, 14(27), 87-106.

Baltaiskalna, D. (2001). Latvijas iedæīotāju lingoistiskā attieksme. Promocijas darba kopsavilkums. Latvijas Universitāte.

Baltiņš, M., y Druviete, I. (2017). Ceļavējš cilvēku ciltij. Valoda sabiedrībā. Rīga: Latviešu valodas aǵentūra.

Comisión Europea. [2012a] (25 de enero de 2021). Informe de la Comisión Europea 2012. https://ec.europa.eu/commission/presscorner/detail/lv/IP_12_990

Comisión Europea. [2012b] (25 de enero de 2021). Eurobarómetro 2012. https:// ec.europa.eu/echo/resources-campaigns/publications/eurobarometer2012

Druviete, I. (1998). Latvijas valodas politika Eiropas Savienības kontekstāa. Latviešu valodas insititūts, LZA Ekonomikas insititūts.

Druviete, I. (2016). Latviešu valoda pasaules sociolingvistisko procesu kontekstā. Latviešu valodas aǵentūra.

Druviete, I., Valdmanis, J. (2010). Language Use in Higher Education Establishments in Latvia. En M.Humar y M.Zagar Karer (Eds.), National Languages in Higher Education (pp. 91-95). Zalozba ZRC.

Encuesta 1. Aptauja par lingvistisko attieksmi pret svešvalodām, t.sk.spāņu valo$d u$, Latvija (Encuesta sobre la actitud lingüuística hacia las lenguas extranjeras, entre ellas, hacia el español, en Letonia). https://docs.google.com/forms/ d/11E8iJHQ0poQILEsP-5khTypPXDKmxlI_nYvI6qdnHHs/edit [Recuperado el 15-04-2021].

Encuesta 2. Aptauja par spāņu valodu Latvijā: apguve un interese par to. (Encuesta sobre el español en Letonia: adquisición e interés por ella). https://docs.google. com/forms/d/1mFtt9iQNAizsO6g8kjjmjdAKDKq2H90kC3sP0tAw3oo/edit [Recuperado el 15-04-2021].

Escoriza Morera, L. (2008). Comentarios de política y planificación lingüústicas. Ariel. Fishman, J. (1995). Sociología del lenguaje. Cátedra.

Harris, J. (2007). Bilingual education and bilingualism in Ireland North and South. International Journal of Bilingual Education and Bilingualism, 10(4), 359-368. https://doi.org/10.2167/beb449.0

Haberland, H. (2011). Local Languages as the Languages of Internationalization. Internationalization and Language Choise. Ibunka komyunikeshon ronshuu/Intercultural Intercultural Communication Review (9), 37-47.

Instituto Cervantes. (3 de mayo de 2021). Informe del Instituto Cervantes 2020. https:// cvc.cervantes.es/lengua/espanol_lengua_viva/pdf/espanol_lengua_viva_2020.pdf

Kibermane, K., y Kḷava, G. (2016). Valodas Latvijā: valsts valoda, valodu prasme, va-

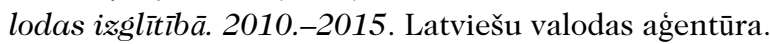

Kristapsone, S. (2014). Zinātniskē pētniecība studiju procesāa. Biznesa augstskola Turība.

Lambert, W. (1964). Social Psychology. Prentice-Hall. 
Alla Placinska / Zenta Liepa

Latvijas Vēstnesis. (5 de mayo de 2021). Latvijas Republikas Augstskolu likums (La Ley sobre la Educación Superior). https://www.vestnesis.lv/ta/id/37967-augstskolu-likums

Legal Acts of the Republic of Latvia. (3 de mayo de 2021). Regulation No.747. Regulations Regarding the State Basic Education Standard and Model Basic Education Programmes. https://likumi.lv/ta/en/en/id/303768

Legal Acts of the Republic of Latvia. (3 de mayo de 2021). Regulation No.416. Regulations Regarding the State General Secondary Education Standard and Model General Secondary Education Programmes. https:/likumi.lv/ta/en/en/ $\mathrm{id} / 309597$

Liepa, Z. (15 de septiembre de 2020). Spanish in Latvia: Language attitude as a factor in the diffusion of the language. Universidad de Letonia. ttps://dspace.lu.lv/dspace/handle/7/52666

Lodares, J. R. (2005). El porvenir del español. Taurus.

López Morales, U. (2004). Sociolingüística. Gredos.

Mayring, F. (2000). Qualitative Content Analysis. Forum Qualitative Sozialforchung/ Forum:Qualitative Social Research, 1 (2), https://doi.org/10.17169/fqs-1.2.1089

Ministerio de Ciencia y Educación de la República de Letonia. (3 de mayo de 2021). Proyecto Skola 2030. https://www.skola2030.lv/lv/skolotajiem/macibu-jomas/ valodas

Medina López, J. (2002). Lenguas en contacto. Arco Libros.

Montoya Ramírez, M. ${ }^{a}$ I. (Ed.). (2005). Enseñanza de la Lengua y Cultura Españolas a extranjeros. Universidad de Granada.

Moreno Fernández, F. (2009). Principios de sociolingüística y sociología del lenguaje. Ariel.

Rozenberga, M. (1999). Spāņu valoda Latvijā. $L U-80$. Konferences referātu tēzes. Latvijas Universitāte,59-60.

Saussure, F, (1931). Cours de linguistique générale. Payot.

Savignon, S. J. (1985). Evaluation of communicative competence: The ACTFL Provisional Proficiency Guidelines. Canadian Modern Language Review (41), 10001007.

Siguan, M. (2001). Bilingüismo y lenguaas en contacto. Alianza Editorial.

Spo Spolsky, B. (2009). Language management. University Press.

Spolskis, B. (2011). Valodas pārvaldība. (Language management). (Traducción del inglés al letón. Traductora I. Druviete). Zinātne.

Yin, R. K. (2009). Case Study Research: Design and Methods. SAGE Publications, Ine. 